\title{
Cultura ciudadana, derechos colectivos y moralidad administrativa
}

Citizen culture, collective rights and administrative morality

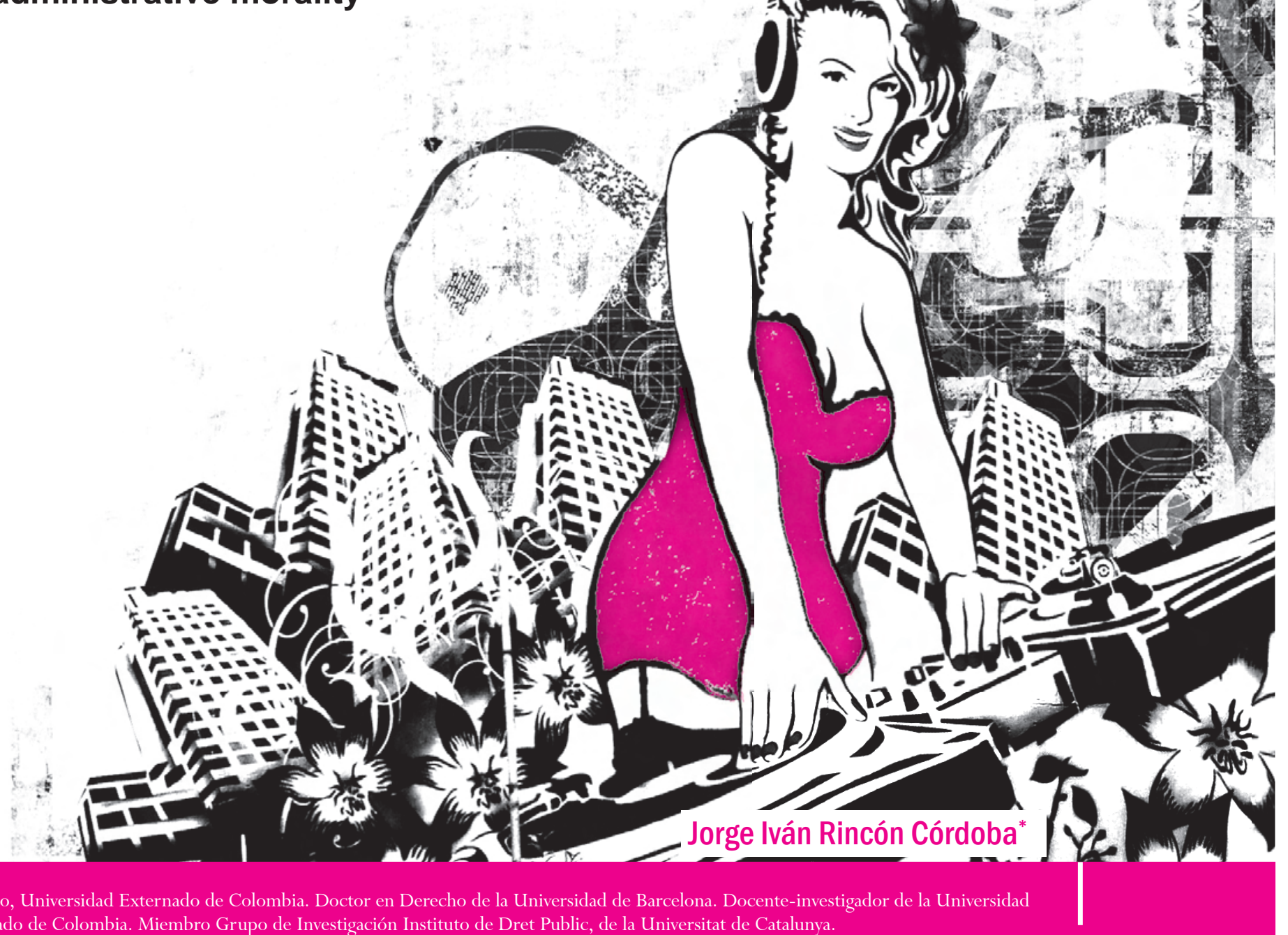


Resumen Este artículo hace parte del trabajo "Metodología pedagógica para la formación de cultura ciudadana a través de los derechos colectivos con énfasis en moralidad administrativa”; en él se pretende, a modo de resumen, identificar aquellos contenidos básicos de los temas de derechos colectivos y moralidad administrativa que son indispensables en la formación escolar de niños, niñas y jóvenes del Distrito, de acuerdo con la reorganización que se ha hecho de la educación en los diversos ciclos académicos. La estructura en que se divide el presente escrito es la siguiente: una delimitación de las nociones que consideramos claves para la formación en cultura ciudadana. En este aparte, más que hablar de conceptos, el lenguaje que se utilizará estará encaminado a señalar acciones y comportamientos que denoten las distintas competencias emocionales y comunicativas que se espera que la población estudiantil adquiera para el ejercicio de una mejor ciudadanía. No haremos un análisis detallado ciclo por ciclo, este trabajo ya se encuentra realizado en el documento entregado al IDEP. Posteriormente, nos detendremos en el modelo pedagógico escogido para la enseñanza de la temática señalada y en la herramienta pedagógica a utilizar.

Palabras claves: cultura ciudadana, derecho colectivo, moralidad administrativa, deberes colectivos, participación ciudadana, principales órganos de la administración distrital, patrimonio público, tributos, pedagogía ciudadana, literatura como herramienta pedagógica.

\section{Abstract}

This paper is part of the project "Pedagogical Methodology for training on citizen culture through collective rights with emphasis in administrative morality". The purpose of this paper is to identify the basic collective rights and administrative morality contents which are indispensable for the school training of children and adolescents of the district according to the reorganization of the education conducted in different academic cycles. In a firs part, a structure of the key notions for the training on citizen culture will be presented. Instead of focusing on the language concepts to be used it will focus on noting actions and behaviors that express the emotional and communicative competences expected from the student population. No detailed analysis on each cycle will be presented; this work is already done in the document delivered to the IDEP. To conclude, there will be an explanation of the pedagogical model chosen for the teaching of the subject and the pedagogical tool to be used.

Keywords: citizen culture, collective right, administrative morality, collective duties, citizen participation, main organs of the district administration, public patrimony, tributes, citizen pedagogy, literature as a pedagogical tool. 


\section{Introducción}

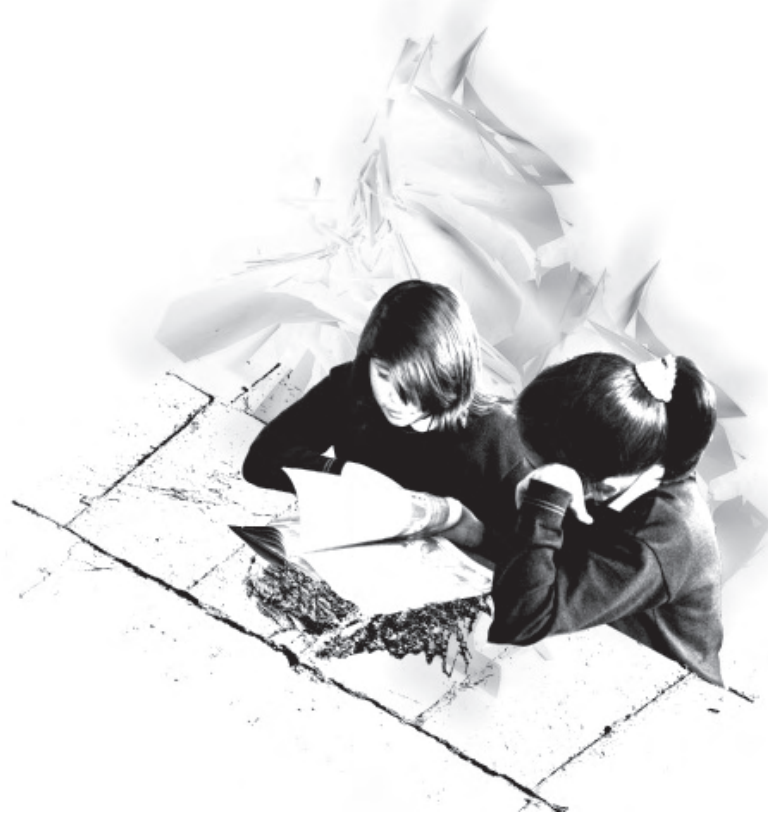

En nuestra sociedad, en la familia pero especialmente en el colegio, el conocimiento de lo público, su cuidado, su organización y administración, no se incluye en la formación de los niños, niñas y jóvenes; esto conlleva a que los estudiantes al terminar su educación escolar desconozcan no sólo los mecanismos o acciones correctas y necesarias para participar en la construcción de lo público sino que, de igual manera, ignoren sus derechos y deberes como ciudadanos y el origen de los recursos públicos indispensables para el funcionamiento de la ciudad.

Es por ello que los derechos colectivos y, en particular, la moralidad administrativa, se convierte en una temática que contribuye a la formación en cultura ciudadana de los estudiantes de colegio, porque sus contenidos permiten ir introduciendo en los alumnos ciertas nociones que, al ser comprendidas e interiorizadas por ellos, generan comportamientos indispensables para la convivencia urbana.
Además, ocasiona que tengan un contacto y una experiencia diferente con su ciudad, así como permite que se involucren con ella, interesándose por la forma como se organizan las autoridades administrativas, como funcionan, y el origen de los recursos que utilizan. Estos aspectos generan en los estudiantes sentido de pertenencia hacia la ciudad, respeto por el patrimonio común y reconocimiento de sus derechos y deberes como ciudadanos, entendiendo a estos últimos no de manera individual sino en una inevitable interrelación con los otros.

Pareciera que lo referenciado fuera un tema estrictamente jurídico y que el conocimiento que de ello se desprende no sea aprehensible por individuos que no tengan una formación en Facultades de derecho o de ciencia de la administración; no obstante, si lo que se pretende es educar en cultura ciudadana y mediante dicha acción (o conjunto de acciones) obtener mejores ciudadanos, debemos partir de una premisa fundamental: los derechos colectivos y por ende la moralidad administrativa hacen parte de aquellos valores consensuados por la colectividad 
para conseguir una mejor y armónica convivencia; sin la comprensión de los mismos no es posible la construcción de una identidad dentro de la ciudad que sea el reflejo de diferentes expresiones del pensamiento, de variadas representaciones artísticas, de diversas formas de concebir ideas y de distintos referentes comunes materiales e inmateriales que identifican al individuo como parte de un grupo.

Vistas así las cosas, la escuela (entendida ésta en un sentido amplio) es el escenario propicio para la adquisición e implementación de "lugares comunes" en la ciudad, dado que lo urbano constituye no sólo un referente geográfico sino de desarrollo de proyectos de vida.
Es en la ciudad, precisamente, en donde confluirán los estudiantes para ejercer sus derechos, aspecto de trascendental importancia para un concepto de ciudadanía que no se circunscribe sólo al ejercicio del sufragio cada cuatro años, sino en actitudes visibles que se traducen en comportamientos de protección, defensa y vigilancia de intereses que desbordan el plano individual, que encuentran como referente inmediato a la comunidad y que se materializan en un medio ambiente adecuado, un correcto disfrute del espacio público, un apersonamiento de la memoria histórica, cultural y arquitectónica, y en conseguir administraciones que cumplan sus funciones motivadas sólo y exclusivamente por el interés general.

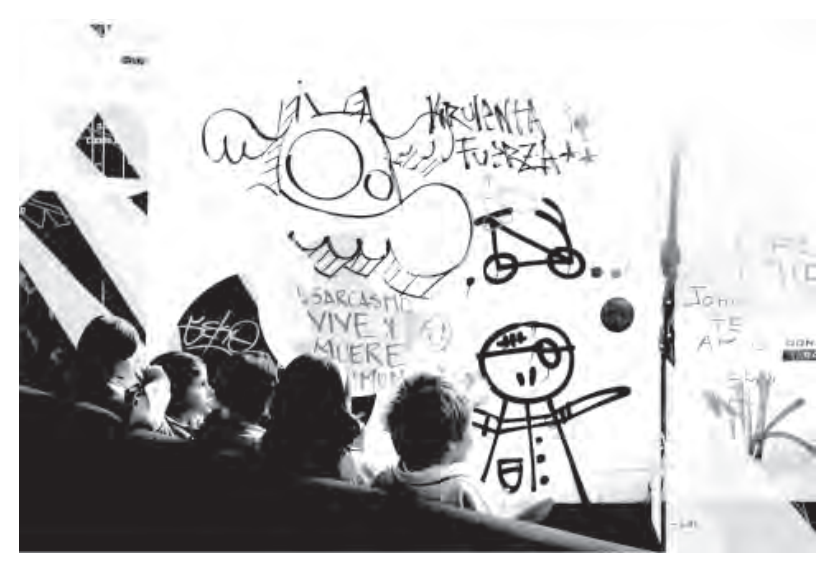




\section{Los contenidos que se desprenden de la noción de derechos colectivos}

Antes de adentrarse en la adquisición de competencias cognitivas, emocionales y comunicativas sobre un derecho colectivo concreto, es indispensable que los alumnos interioricen cuál es el concepto de derechos de tercera generación e identifiquen los elementos que los componen. En otras palabras, no es posible dimensionar correctamente qué es y cómo se constata la moralidad administrativa dentro de la ciudad sin antes detenerse en la definición de "derecho colectivo" que la enmarca.

Así las cosas, el punto de partida para maestros y educadores es el de no elaboración de discursos absolutos, dado que el tema de los derechos colectivos no es estático sino que obedece a un proceso dinámico que admite lecturas diversas y heterogéneas (Pérez Luño, 2002: 94).

Partiendo del anterior presupuesto, es inevitable que hagamos una segunda advertencia: cada uno de los contenidos que se desprenden del término "derechos colectivos" no debe enseñarse partiendo de una metodología de transmisión tradicional de conocimientos; al hablar de derechos nos introducimos en un discurso de valores consensuados, y es precisamente este el punto de partida: las conclusiones deben ser el resultado de la interacción entre docente y alumnos, donde el primero ha de convertirse en un mediador, en un potenciador de inquietudes.

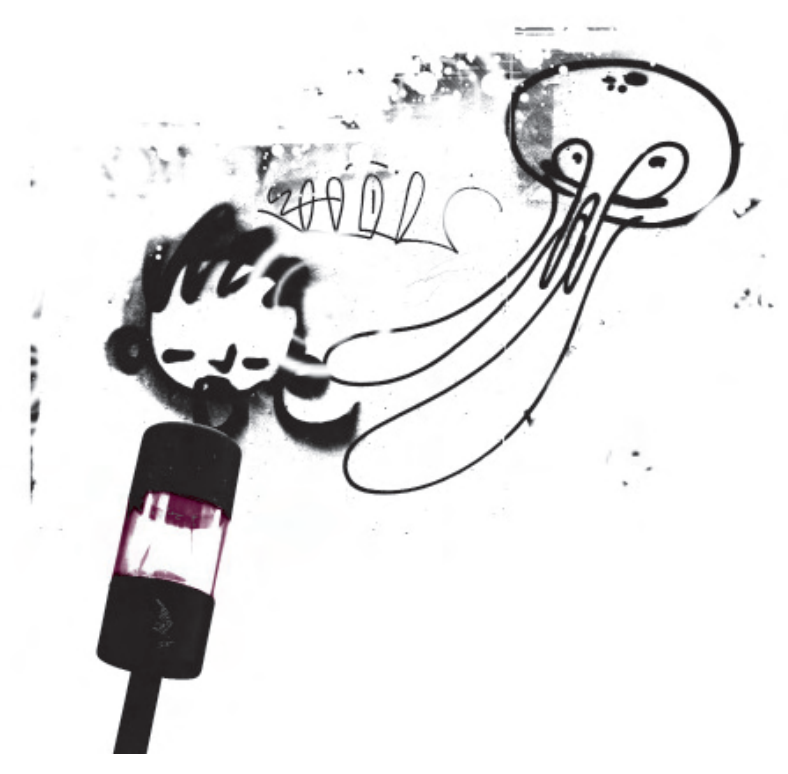

Dado que los derechos colectivos obedecen a un proceso de continua evolución, son el resultado de una realidad histórica (Peces-Barba, 1998), precisan de contextualizarse de acuerdo con la realidad que vive el estudiante para hacerle entender que la existencia de estos nuevos derechos obedece precisamente a los hechos que él constata en su cotidianidad: la constante interacción con la tecnología, la diversidad cultural que subyace en la ciudad, los problemas medioambientales y el efecto extensivo de la contaminación. 


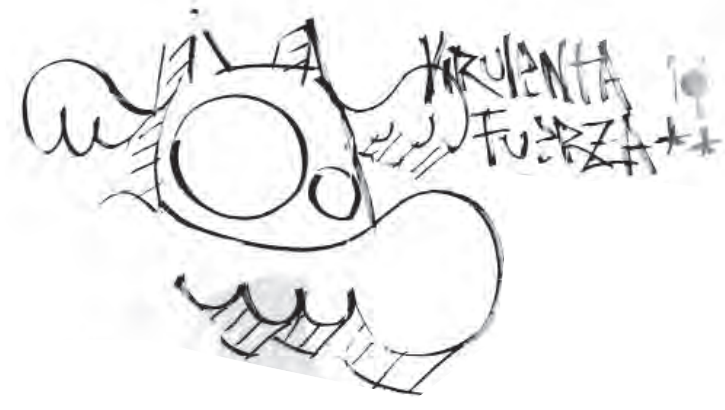

Sólo si se comprende el entorno es posible llegar a la generación de un primer contenido que, a su vez, se traduce en comportamientos concretos; la búsqueda de soluciones a los problemas que se generan por factores como los indicados atrás exige que cada ciudadano identifique intereses que ya no se explican desde el plano de lo individual; dicho plano es trascendido por la necesidad de inclusión de los otros, por el deseo y conciencia de pertenecer a un colectivo.

Surge, entonces, un concepto que debe ser una constante en todos y cada uno de los ciclos académicos: la solidaridad, que debe traducirse en compromisos visibles de cada sujeto con su comunidad. A la individualidad propia de los clásicos derechos de libertad, o a la igualdad material propia de los llamados derechos sociales, se suma la identificación de valores comunes como un presupuesto inevitable para comprender en su justa dimensión el valor de "dignidad humana”, traducible en un mejoramiento ostensible de la calidad de vida de las poblaciones (Rincón Córdoba, 2004: 499).

Un segundo componente se desprende de lo dicho hasta aquí: niños y jóvenes deben tener presente que la comprensión del concepto derecho colectivo es trascendental, porque la afectación de derechos como los del medio ambiente o el espacio público tienen una capacidad de generar daños a escalas insospechadas, muchas veces no reparables, y que, por tanto, no sólo los afecta a ellos en su presente sino también a las generaciones futuras (Beck, 2002).

Esta capacidad de generar daños irremediables pone en alerta a la comunidad, la cual precisa de replantearse su responsabilidad en el tratar de evitar comportamientos lesivos, y acá nuevamente se subraya la necesidad de construcción de un lenguaje que deje de lado el "yo" (sin renunciar a él) para detenerse en el "nosotros" (Colaço Antunes, 1991: 417).

Si lo dicho hasta aquí es aprehendido por el estudiante, es posible dar un paso más. No sólo basta la identificación de los factores señalados, adicionalmente se requiere que el niño, la niña o el joven comprendan que su relación con el sector público no es mediata sino inmediata, por tanto, sólo es posible la protección de aquello considerado común si se asumen actitudes concretas: utilización de instancias de participación (mecanismos como la solicitud de documentos, preguntas a través de derechos de petición, inclusión en procedimientos de 


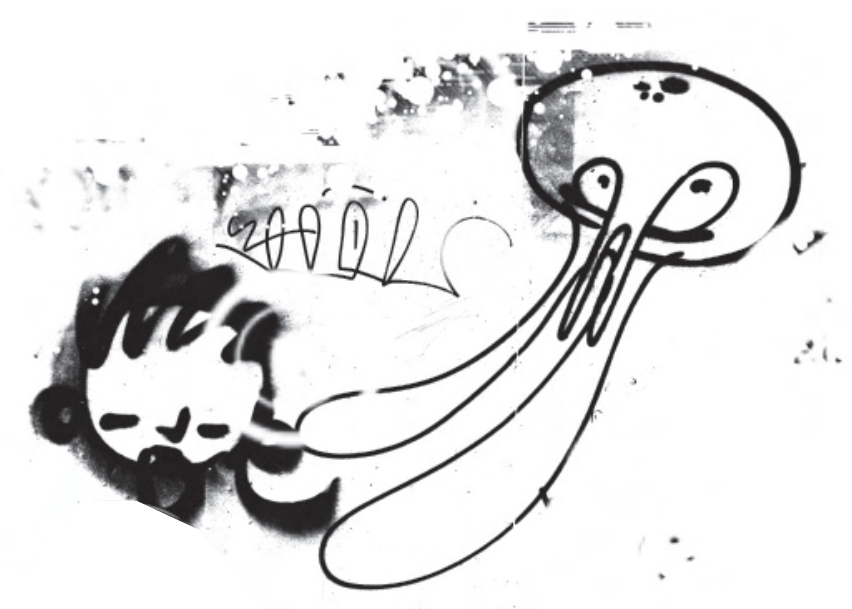

las autoridades administrativas en la toma de decisiones, etc.), la inserción en organizaciones administrativas, la vigilancia directa mediante asociaciones y veedurías ciudadanas, el ejercicio de acciones judiciales, etc. (Pisciotti Cubillos, 2001). Adicionalmente, si de la inclusión en un entorno colectivo se trata, nuevamente una luz roja se debe poner en la labor que adelantan los docentes: lo colectivo conlleva indiscutiblemente diversidad, rechaza lo homogéneo. Por tanto, un contenido indiscutible es el de "interculturalidad", concepto que no se restringe sólo a la aceptación de aquello que es diferente sino a la interacción; solo de este modo es posible el consenso del que hablamos, sólo así cada individuo incluirá al otro dentro de aquello que considera común, aunque lo común parta de lo diverso (De Asís, 2005: 65).

De esta forma, y a modo de recapitulación, durante los diversos ciclos académicos los niños y jóvenes deben adquirir los siguientes conocimientos, comunicarlos y traducirlos en acciones:

1. La importancia de ser solidarios en el entorno comunitario, puesto que la protección de lo colectivo demanda de ellos colaboración directa y asunción de responsabilidades, entre ellas el cuidado de aquello que se comparte.
2. La identificación de intereses comunes dentro de la ciudad es un reflejo mismo de identidad, dado que la misma no puede construirse de manera aislada respecto a la realidad circundante.

3. La protección de derechos colectivos conlleva en muchos casos una renuncia a derechos individuales; para asegurar el interés de todos es inevitable tener que ser capaces de renunciar a intereses que partan exclusivamente de la individualidad. Ello no significa renuncia del espacio de autodeterminación, sino inclusión dentro del mismo de nuevos valores.

4. La identificación de dichos intereses colectivos en la manera como se estructura administrativamente la ciudad; la autoridad pública vista no como el único responsable del cuidado de lo colectivo sino como una instancia de referencia a través de la cual confluyen los comportamientos de la ciudadanía.

5. El conocimiento de los diferentes instrumentos de participación democrática como canales de comunicación entre la autoridad y los ciudadanos para hacer posible una mejor convivencia a través de la protección y defensa de intereses colectivos. 


\section{Los contenidos que se desprenden de la noción de "moralidad administrativa"}

De todos los derechos colectivos, la moralidad administrativa resulta ser el de más difícil definición. Aun así, es posible circunscribirla a un determinado modo de comportarse de la autoridad administrativa. Como podemos notar, nos encontramos frente a un concepto que se refiere exclusivamente al ámbito interno, al cómo funcionan y desempeñan competencias aquellos que adelantan actividades administrativas.

Aclarado el anterior aspecto, nuevamente se requiere hacer una advertencia: moralidad administrativa no es equivalente a moral. Es cierto que la escuela se convierte en un referente ético para el estudiante y que dentro de la misma no sólo se transmiten conocimientos sino además reglas sociales de comportamiento. Sin embargo, volvemos a insistir: los valores comunes deben ser producto del consenso, la moral es un discurso que pertenece al ámbito interno de cada individuo, y por lo tanto, parte de criterios absolutos. Si lo que se pretende es educar en la construcción de mejores ciudadanos, el discurso debe volverse externo, esto es, que lo relevante debe ser única y exclusivamente aquello que puede llegar a tener una incidencia significativa en la construcción de un modelo de sociedad. No se trata de elaborar discursos sobre lo bueno o lo malo sino de implementar instancias en las que se llegue a acuerdos sobre aquello que es preponderante para coexistir en armonía con los otros.

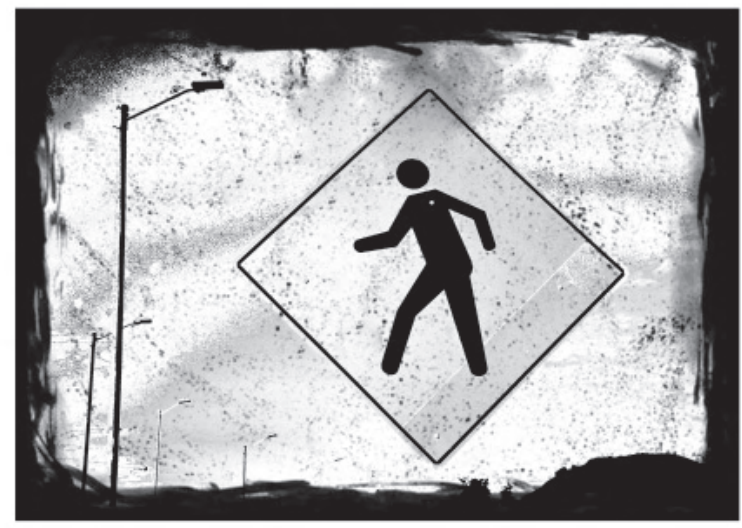

En la anterior construcción se ubican los derechos colectivos y en particular el derecho a la moralidad administrativa. No obstante, en este plano ocurre algo bastante curioso, los valores representados no sólo importan a la sociedad sino además al Derecho. Así, tanto en el plano social como jurídico resulta indispensable que la actividad administrativa se adelante de tal forma que no dé lugar a dudas razonables de los ciudadanos sobre la existencia de comportamientos o conductas contrarias a la honestidad y pulcritud en el manejo de lo público. 


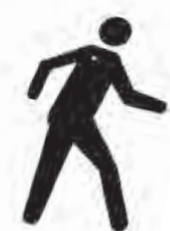

Tal como advertimos, es un concepto etéreo y que parece de difícil trasmisión en un lenguaje sencillo que lo haga aprehensible por estudiantes de colegio. Empero, ello no es así, dado que si se identifican valores colectivos enmarcados en derechos, aquello que debe ser interiorizado por el niño o joven es que las instancias administrativas sólo pueden actuar persiguiendo la satisfacción de estos intereses, pues si a este objetivo se antepone el querer individual de quien administra, la conducta, claramente contraria de lo que debe entenderse por ética de lo público, puede incluso catalogarse como un caso concreto de corrupción.
De la definición dada puede extraerse otra posible consecuencia: si la moralidad administrativa se traduce en un derecho a actuar de acuerdo con principios como la transparencia y la objetividad, y sobre todo basada en la persecución exclusiva del interés de la comunidad, la no vigilancia y el desinterés de los individuos puede generar la afectación de otros derechos colectivos como, por ejemplo, el patrimonio público. Estamos, entonces, en presencia de un presupuesto indispensable para el disfrute de intereses grupales.

Dicho lo anterior, es necesario hacer una salvedad. La idea de derecho exigible únicamente a la autoridad administrativa no puede desviar la atención de aquello que resulta relevante para el individuo: la asunción de deberes como condicionamiento para el disfrute de derechos. Solo si se incorpora la idea de derechosdeberes podemos conseguir ciudadanos que se interesen por el bienestar de los demás, asuman posiciones de vigilancia constante, y que en el caso de incorporarse en instancias de decisión obrarán siempre en pro de la comunidad. En otras palabras, los individuos que demandan las nuevas sociedades son aquellos que no son meros observadores sino que se convierten en partícipes y gestores de lo público y coadyuvan en su materialización.

Los elementos hasta aquí enunciados nos permiten entrar a identificar dichos deberes y responsabilidades y traducirlos, tal como hicimos con el concepto de derechos colectivos, en comportamientos que se desprenden de la adquisición de competencias cognitivas, emocionales y comunicativas por parte de los niños, niñas y jóvenes del Distrito: 
1. La solidaridad nuevamente es el concepto a asumir, pero en esta ocasión el estudiante no sólo debe conformarse con incluir dentro de su individualidad los intereses de los otros, adicionalmente debe interesarse en todo momento por el manejo de lo público, asumirlo como algo que le compete, como algo propio de su incumbencia. Ser solidario no sólo implica tener una ética privada que permita actuar sin afectar a otros, conlleva la inclusión de una ética de lo público que demanda mucho más: el comportarse de determinada forma (De Asís, 2005: 49).

2. Dentro de ese comportarse de determinada manera se encuentra, precisamente, el conocer cómo se conforma y organiza lo público. Por ende, los jóvenes, cuando terminen su formación escolar, deben saber cómo se estructura la administración de la ciudad, cuáles son las autoridades principales, qué clase de actividades gestionan y cuál es la misión encomendada a cada una de ellas. De igual manera, conocer el sistema tributario, no en sus detalles técnicos, pero sí en cuanto a cuáles son las principales fuentes de ingresos a través de las cuales se hace posible el gasto público. Estos dos aspectos son determinantes, porque si se quieren administraciones que actúen de acuerdo con intereses comunitarios habrá que informarse primero de quiénes tienen a su cargo el cuidado de los mismos y cuáles son los medios financieros que poseen. En el último caso, si moralidad y patrimonio van de la mano, un manejo ético del segundo conlleva inevitablemente el conocimiento de los diferentes elementos que lo conforman.
3. Una vez se conoce cómo funciona el sector público, surge un deber que se sitúa en un tercer nivel: cada individuo puede hacer uso de los cauces de participación activa existentes para conseguir actuaciones administrativas ajustadas a los intereses generales. Así, se pueden identificar tres derechos concretos que enmarcan el comportamiento al que hacemos referencia: a) el control de la actividad administrativa mediante el acceso a la información que reposa en las diferentes dependencias públicas; b) la posibilidad de participar en la toma de decisiones administrativas a través de la presentación de solicitudes respetuosas, y c) la posibilidad de utilizar las acciones populares en aquellos eventos en que se constaten comportamientos contrarios al derecho colectivo o a la moralidad administrativa.

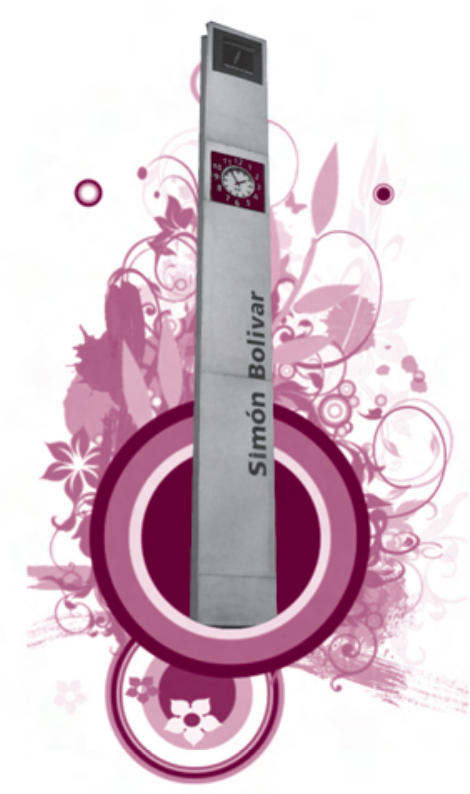




\section{Los contenidos que se desprenden del conocimiento de la estructura administrativa del Distrito}

La forma como el derecho colectivo a la moralidad administrativa encuentra su reflejo en el Distrito es precisamente el deber de conocimiento de lo público que radica en cabeza de todo ciudadano. En la actualidad la información es valiosa, por lo tanto, el dominio de la misma es el primer paso para utilizar de forma efectiva cauces de participación democrática o instrumentos judiciales para la protección de intereses colectivos. De aquí se deduce que los contenidos que se desprenden del conocimiento de la estructura sean los que a continuación se enumeran:

1. Los estudiantes de colegio deben comprender que la forma como se estructura administrativamente su ciudad obedece a las respuestas que dentro de la misma se encuentran para enfrentar realidades concretas y diversas.

2. Esta originalidad en el diseño administrativo no es contrario a la identificación de unos organismos básicos que caracterizan constitucional y legalmente a todo municipio o ciudad en Colombia. En efecto, los niños y jóvenes han de saber que el Concejo Distrital es la principal instancia de representación popular, un escenario colegiado en el que mediante la deliberación se toman las decisiones más importantes

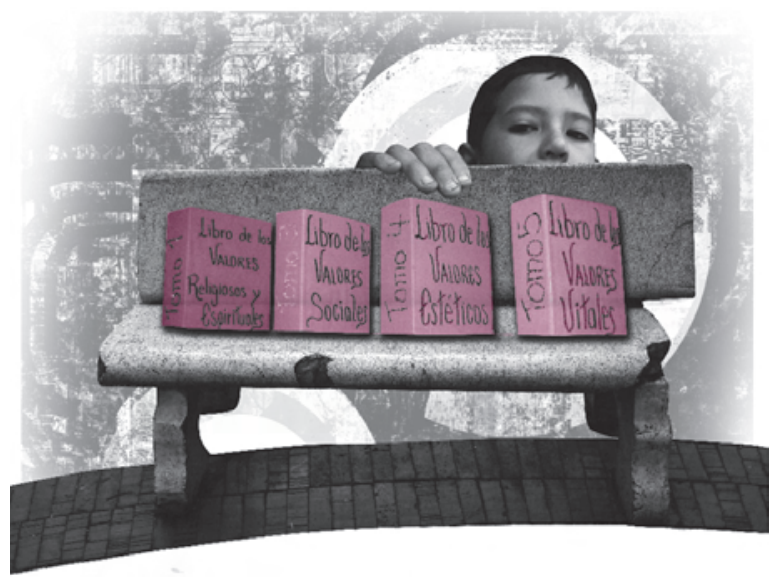

que afectan la vida de los bogotanos. Adicionalmente, tener en el alcalde un referente político de gobierno; en él también se presenta el fenómeno representativo (es elegido mediante votación popular), pero, a diferencia del órgano colegiado, su característica principal es la de diseñar las políticas públicas dentro del ámbito distrital y sobre todo aplicar las normas mediante la dirección de aquellos organismos que se encargan de la prestación de servicios y de la generación de obras públicas.

3. Otro rasgo particular de la ciudad de Bogotá es la denominada descentralización territorial dentro del Distrito. Es precisamente este aspecto el que 


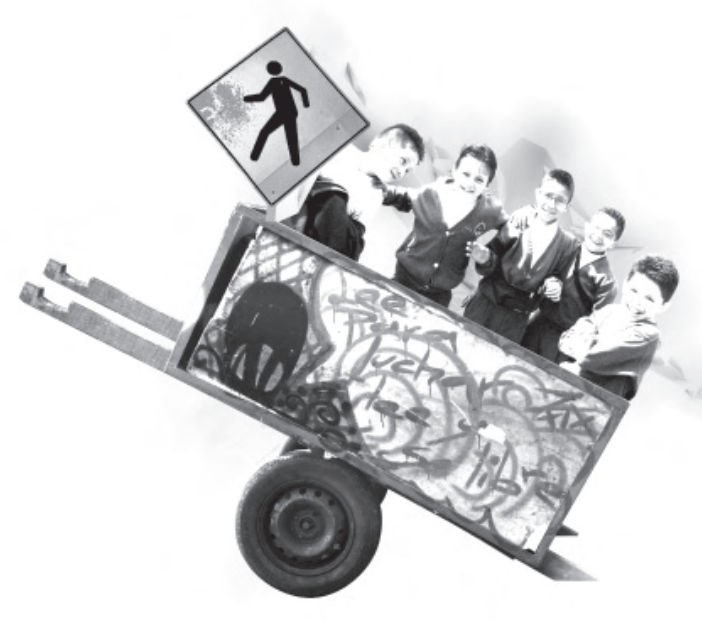

£

debe constituir un referente inmediato para el estudiante; dado que forma parte de una localidad, le es pertinente identificar las instancias de poder más cercanas, refiriéndonos a las juntas administradoras locales y a los alcaldes locales.

4. De igual modo, resulta esencial para asegurar administraciones públicas que obren de acuerdo con los intereses generales, tener conocimiento de los llamados organismos de control. Mediante los mismos, los cauces de participación democrática se conjugan con mecanismos institucionales que se encargan de vigilar el actuar público. Así, como conceptos básicos para aprehender encontramos: a) el conocimiento sobre las funciones de la Personería Distrital, ante todo como ministerio público y principal difusor de los derechos humanos; b) el conocimiento relativo a las funciones de la Veeduría Distrital como órgano encargado de la vigilancia de la actuación de las autoridades administrativas distritales, y c) el conocimiento atinente a las funciones de la Contraloría, principalmente sobre su labor de supervisar si los recursos públicos son utilizados y gestionados de forma correcta.
5. Adicionalmente, el estudiante como futuro ciudadano debe identificar los sectores administrativos a través de los cuales se adelantan las competencias del Distrito. En consecuencia, no sólo ha de saber cuáles organismos se encargan de la dirección de dichos sectores (las denominadas Secretarías de Gobierno) sino además tener conocimiento sobre aquellas entidades que se encargan de la prestación de servicios públicos concretos (los establecimientos públicos), el adelanto de programas o funciones específicas de las instancias de dirección (unidades administrativas especiales), de las que desarrollan actividades consideradas industriales y comerciales (empresas industriales y comerciales del Estado, sociedades de economía mixta) o de las que prestan servicios públicos domiciliarios o de salud (empresas oficiales de servicios públicos, empresas sociales del Estado). Sólo de esta manera se puede tener claro quiénes son los responsables de los distintos intereses colectivos y es posible su supervisión directa, así como la exigibilidad de responsabilidades. 


\section{Los contenidos que se desprenden del denominado patrimonio público}

En el deber de todo ciudadano de conocer lo público se encuentra, precisamente, lo relativo a la conformación del denominado patrimonio público. Este derecho colectivo es traído a colación por su íntima relación con el derecho a la moralidad administrativa. El contenido del mismo se puede sintetizar en el derecho que tiene la colectividad a que los bienes y recursos en manos de las autoridades públicas sean gestionados de manera adecuada. Recordemos que hablar de moralidad es igual a decir que las competencias administrativas se ejercen persiguiendo exclusivamente intereses generales; si ello es así, resulta contrario a este interés colectivo que el manejo de los recursos públicos no se ajuste a las disposiciones de carácter presupuestal, esto es, que no solventen las necesidades sociales que justifican su existencia.

Adicionalmente, comprender el concepto de patrimonio público implica tener presente que dentro del mismo se abarcan diversas clases de elementos. En primer lugar, un elemento de carácter objetivo, dado que no se restringe a los bienes muebles o inmuebles, sino que en la noción analizada se incluye cualquier activo o derecho reconocido a favor del Distrito. De igual modo, encontramos un elemento subjetivo, determinado por la necesidad de identificar como titular a un ente público (el Distrito o cualquiera de sus entidades descentralizadas).
Por último, el elemento más importante, la finalidad a la que sirve: hacer posible el cumplimiento de las funciones y competencias que tienen asignadas las distintas instancias públicas.

Por contera, de lo dicho en el párrafo precedente se desprende que la actitud de vigilancia sobre los componentes del patrimonio público sólo es posible si se es consciente de los elementos que lo conforman. Por ello, un primer contenido es el atinente precisamente a la diferenciación entre bienes de uso público, bienes fiscales y bienes presupuestales. Respecto de los primeros, la característica básica es su destinación al uso general de la colectividad, y frente a ellos la administración adelanta ante todo una labor de gestión. Como ejemplo podemos encontrar bienes tales como reservas naturales, ríos, calles, plazas, puentes, etc.

A diferencia de los anteriores, los bienes fiscales se caracterizan, precisamente, no por estar destinados al uso común sino por hacer posible el funcionamiento de las entidades administrativas; sobre los mismos la administración actúa como propietaria. Ejemplo de ellos lo constituyen los edificios que sirven de sede a las entidades públicas. 
Por último, se encuentran aquellos bienes denominados presupuestales, constituidos por recursos obtenidos a través del ejercicio de la denominada potestad impositiva, es decir, recursos que se obtienen mediante la recaudación de impuestos por el Distrito. En estos últimos nos detendremos, dado que las dos primeras categorías explicadas pueden enmarcarse dentro de los trabajos adelantados en el proyecto "Innovación pedagógica para la formación de cultura ciudadana”, específicamente aquellos que se ocupan del medio ambiente, del espacio público, y del patrimonio histórico, cultural y arquitectónico.

Así, un segundo contenido es el referente al del "deber de contribuir", de tal manera que cuando se hable de tributos el estudiante no piense apenas en la imposición hecha desde las instancias de poder, sino que además sea consciente de que dicha imposición se justifica, precisamente, porque sólo a través de la colaboración de todos los individuos que se encuentran en el territorio del Distrito es posible dar cobertura a imperativos sociales como la salud, la educación, la recreación, la protección del medio ambiente, la correcta gestión del espacio público, etc.

Esto es lo que se denomina el efecto motivador del tributo, y que nos sirve para explicitar la adquisición por parte del estudiante de otras competencias que se relacionan directamente con el mismo.
En primer lugar, el niño o joven deben tener presente que ningún impuesto puede llegar a afectar una actividad económica. Siempre que se procede a su creación la autoridad analiza los posibles efectos negativos que pueden desprenderse del mismo (principio de universalidad).

En segundo término, el ciudadano que se quiere obtener ha de tener presente que el pago de impuestos no conlleva una contraprestación directa, dado que quien contribuye lo hace para obtener el bienestar general de la colectividad (teoría del beneficio). Así mismo, y en consonancia con lo anterior, vuelve a manifestarse el principio de solidaridad, de modo tal que aquellos que más tienen deben contribuir en mayor medida a la satisfacción de necesidades de sectores de la población que se encuentren en desventaja (teoría de la capacidad de pago).

Por último, una persona formada en cultura ciudadana requiere asumir un comportamiento del cual se desprenda que cumple sus obligaciones tributarias sin interponer obstáculos, pero a este último peldaño únicamente se arriba si se han recorrido los escalones precedentes. Si el estudiante ya ha comprendido la importancia del contribuir, porque tiene los conceptos, los traduce en lenguaje y los comunica, y además cree en la importancia de lo aprehendido, la impartición de contenidos referentes a la delimitación de los varios impuestos del Distrito es una labor complementaria que es necesario detener sobre todo en la delimitación de aquellos hechos que dan lugar a su cobro y recaudo: el adelanto de una actividad industrial o comercial, el ser propietario o poseedor de un vehículo, la venta de loterías foráneas en el Distrito, etc. 


\section{El modelo pedagógico urbano escogido: el rescate de un modelo basado en la construcción de experiencias}

Ya vistos los contenidos sobre los cuales se cimienta la formación en cultura ciudadana de los estudiantes del Distrito, pasamos ahora a delimitar el modelo pedagógico seleccionado para proceder a la enseñanza de los mismos y a la consecución de competencias cognitivas, emocionales y comunicativas en los niños y jóvenes en sus ciclos académicos. Por esto, un primer aspecto a subrayar es precisamente el identificar que lo urbano se caracteriza porque dentro de sí coexisten diferentes realidades que se desarrollan en distintos planos (la escuela, el barrio, la ciudad), comprenden variadas formas de concebir el mundo (indígenas, afrocolombianos, mestizos y extranjeros), abarcan diversas posturas frente a las problemáticas sociales (estratos económicos), o expresan múltiples ideologías religiosas y políticas, etc.

El escenario descrito requiere de un modelo pedagógico que asuma la enseñanza como un proceso social y de formación del ser humano. Si ello es así, los contenidos impartidos no deben obedecer a un paradigma basado en una relación vertical entre alumno y profesor. Al contrario, el papel del maestro debe ser el de desarrollar en el alumno autonomía, formación de criterios propios y ejercicio de libertad de manera responsable, es decir, teniendo en cuenta a los otros.

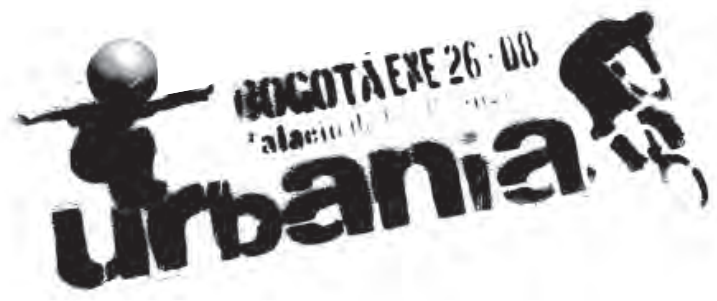

En este contexto, los derechos colectivos requieren de ser mostrados a los alumnos como parte de su cotidianidad, pues sólo así asumirán fácilmente las responsabilidades que de los mismos se desprenden. No obstante, la asunción de dichas responsabilidades debe ser la respuesta a la capacidad de discernimiento de los estudiantes, capacidad que necesita ser desarrollada en el interior de las aulas de clase. El colegio tiene que ser el lugar propicio para que se construyan proyectos de vida individuales y grupales, y en dicha elaboración no puede omitirse la historia de los otros: al contrario, es ineludible incluirlos para así comprender que la sociedad es la sumatoria de muchas y variadas vivencias. 


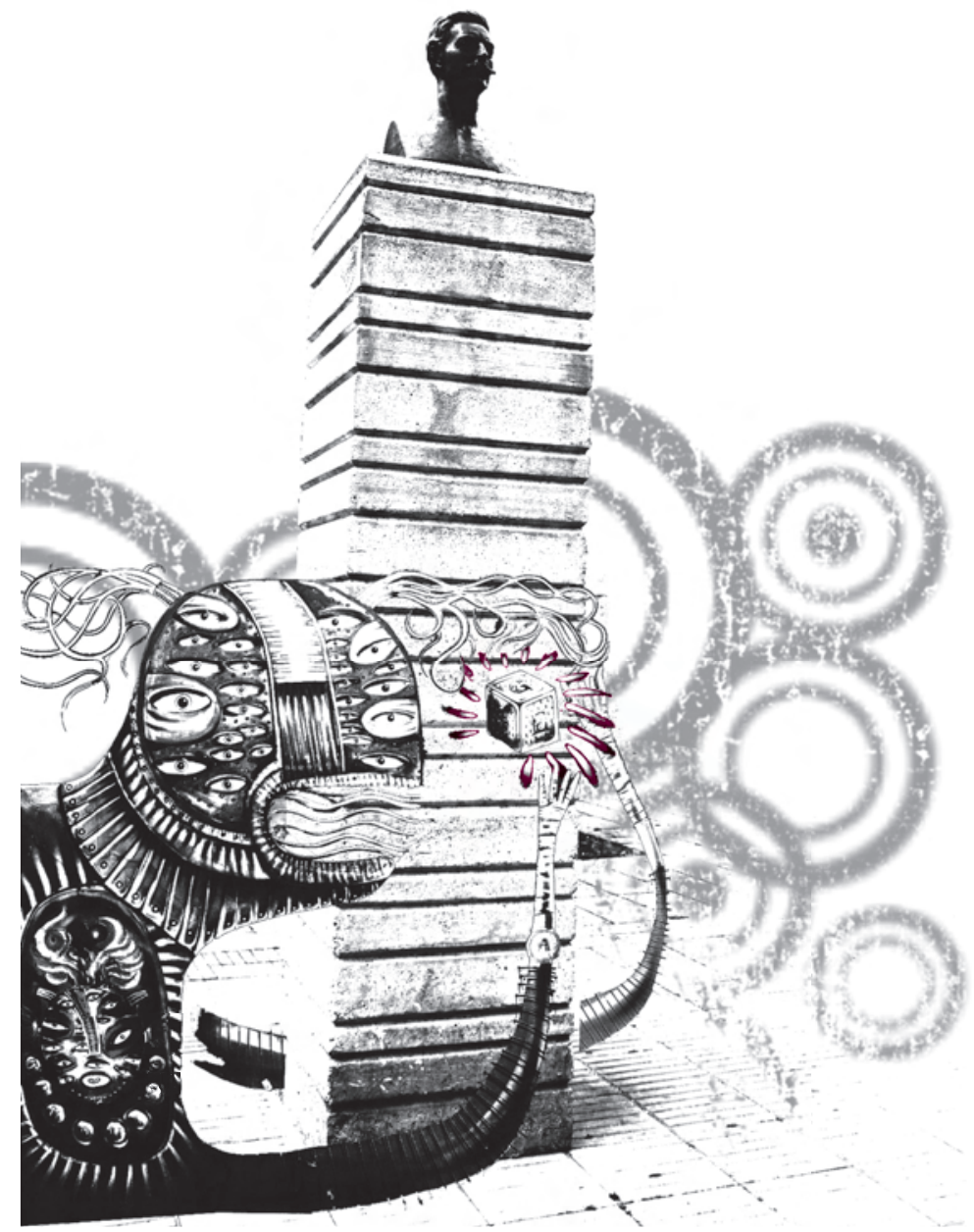

En el proyecto de sociedad por construir los derechos y deberes ocupan un lugar preferente, dado que de ellos depende la convivencia en armonía, y por ello la interiorización de los mismos desemboca en comportamientos de respeto y cuidado. El paradigma o modelo deseado sólo se puede alcanzar en un esquema en el que la relación alumno-maestro sea el eje estructurador del proceso formativo del primero. De esta forma, el diálogo respetuoso, la construcción conjunta de saberes, y el cuestionamiento constante de la realidad, son los parámetros a seguir. La relación aquí descrita requiere ser paritaria, sin que ello conlleve ausencia de posicionamiento del profesor, quien con su actuar constituye un referente inmediato para sus estudiantes. Si el diálogo constante es el método, la escuela debe propiciar los espacios indispensables para que los alumnos expresen sus sentimientos e inquietudes. Si existe una interrelación constante se puede conseguir el consenso mediante la legitimación del otro, a través del mutuo reconocimiento y el intercambio respetuoso de posiciones.

El diálogo exige unas habilidades específicas del educador, especialmente el desarrollo de una mayor intuición y una mayor capacidad de asimilación de las diferencias existentes entre sus alumnos. Él ya no se debe conformar con la faceta de simple transmisor de conocimientos, ahora es el determinante en la construcción de procesos mentales. Por consiguiente, se hace relevante que el maestro rescate la llamada mayéutica socrática como instrumento que posibilita discutir, construir y contextualizar en clase conceptos tales como solidaridad, convivencia, derechos, deberes, etc., por medio de la reflexión y la generación de propuestas que provengan de los alumnos y que contengan un referente inmediato sobre sus entornos más cercanos: el colegio y el barrio, para que luego los comportamientos generados tengan un reflejo en escenarios más amplios: la localidad y la ciudad. 


\section{Las herramientas pedagógicas}

Finalmente, si el método se sustenta en el diálogo y en el intercambio constante de pareceres, las herramientas pedagógicas que pueden potenciar la aprehensión de los contenidos señalados son la literatura y la escritura. Mediante estos dos instrumentos se posibilita la reflexión, se propicia la autoexploración y se fomenta el autoconocimiento.

La selección de textos adecuados permite que el alumno se ubique en otros contextos, conozca diferentes culturas, aprenda a aceptar diversas posiciones sobre una misma problemática y llegue a conclusiones extrayendo enseñanzas concretas. Si este proceso se complementa con el escribir, aquello que de la lectura llama la atención y genera controversia es revisado y permite la generación de nuevos saberes. La labor del maestro es en extremo importante, él debe seleccionar los textos, pero esta tarea necesita realizarla de tal forma que no pretenda inducir a conclusiones. Una vez escogido el texto, su papel es el de facilitador de las variadas visiones que se generen y moderador en los valores que se construyan y se refuercen mediante el debate generado. Por medio de la literatura se pueden interiorizar conceptos como solidaridad, respeto, convivencia, interculturalidad, pluralismo, derecho y deber, interés colectivo, entre otros.
La herramienta pedagógica escogida debe complementarse con otros dos instrumentos que consideramos indispensables para la interiorización de los contenidos que se desprenden del deber de conocimiento de lo público: en primer lugar, la necesidad de conocer las entidades principales del Distrito, hablar con sus representantes, que se les narre la importancia de ellas en la defensa de los derechos colectivos. La experiencia de otros es un referente necesario porque únicamente se siente como propio aquello que se conoce; por ello, las "excursiones" o el "sacar la clase de los muros del colegio" es indispensable.

En segundo y último lugar, el conocimiento y utilización de los denominados mecanismos de participación se pueden implementar mediante las distintas instancias de representación y participación propias del gobierno escolar. De esa forma, además de seleccionar a los voceros de la comunidad académica (representantes del curso, personeros, representantes ante el Consejo Directivo, etc.) se facilita trasplantar mecanismos como la posibilidad de pedir información a dichas instancias representativas, la elevación de solicitudes respetuosas, e incluso la conformación de veedurías para constatar el correcto funcionamiento de las múltiples instancias de gobierno. 


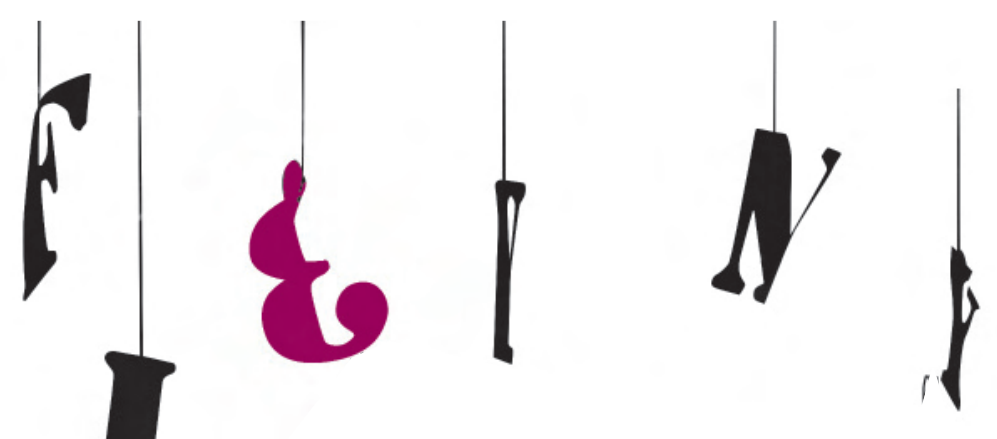

\section{Bibliografía}

Albi, F. Tratado de los modos de gestión de las corporaciones locales, Madrid, Aguilar, 1960.

Alexy, R. "Tres escritos sobre los derechos fundamentales y la teoría de los principios", en Serie de teoría jurídica y filosofía del Derecho, no 28, Bogotá, Universidad Externado de Colombia, 2003.

Alonso, C.; Cruz, A.; Lizama, L. "La inteligencia emocional, implicaciones", en La educación y el mundo del trabajo, Bogotá, El Búho, 2002.

Alonso Velasco, J. M. "Ciudad y espacios verdes". Monografías de vivienda, arquitectura y urbanismo, Madrid, Ministerio de la Vivienda, Servicio Central de Publicaciones, 1971.

Amatucci, A.; González García, E. “El concepto de tributo”, en Tratado de Derecho Tributario, Bogotá, Editorial Temis, 2001.

Ansuategui Roig, F. "De los derechos y el Estado de Derecho. Aportaciones a una teoría jurídica de los derechos", en Serie de teoría jurídica y filosófica del Derecho, no 47, Bogotá, Universidad Externado de Colombia, 2007.

Asensio, J. M. Una educación para el diálogo, Barcelona, Paidós, 2004.

Beck, U. La sociedad del riesgo global, Madrid, Siglo XXI, 2002.

Borowki, M. "La estructura de los derechos fundamentales", en Serie de Teoría Jurídica y Filosofía del Derecho, no 25, Bogotá, Universidad Externado de Colombia, 2003.

Bujosa Vadell, L. La protección jurisdiccional de los intereses de grupo, Barcelona, Editorial Bosch, 1995.

Caijao, F. “Lo esencial no es pasar el año”, en El Tiempo, Bogotá, 14 de octubre de 2008.

Casabene De Luna, S. "Nociones fundamentales sobre el derecho del medio ambiente", en Lecturas sobre derecho del medio ambiente, t. I, Bogotá, Universidad Externado de Colombia, 2000.

Cassese, S. Las bases constitucionales del Derecho Administrativo, Madrid, Ministerio de Administraciones Públicas, 1994.

Chiroque Chunga, S. "Investigación sobre y para la innovación educativa y pedagógica", en Encuentro de investigadores e innovadores en educación, Santiago de Chile, Convenio Andrés Bello, 1996.

Colaço Antunes, L. F. "Los intereses difusos: ubicación constitucional; tutela jurisdiccional y 'acción popular de masas' (en torno a la revisión de la Constitución portuguesa de 8 de julio de 1989)”, en Revista de Administración Pública, no 124, Madrid, Centro de Estudios Constitucionales, 1991. 
Colmeiro, M. Derecho Administrativo español, t. I, Madrid, Imprenta y Librería de Eduardo Martínez, 1876.

Cosculluela Montaner, L. Manual de Derecho Administrativo, t. I, Madrid, Cívitas, 2007.

De Asís, R. "Cuestiones de derechos", en Serie de teoría jurídica y filosófica del Derecho, no 37, Bogotá, Universidad Externado de Colombia, 2005.

Debesse, M. Historia de la Pedagogía, Barcelona, Oikos, 1973.

Dilthey, G. Historia de la Pedagogía, Buenos Aires, Losada, 1968.

Espinel Vallejo, M. "La educación y la convivencia ciudadana", en Encuentros de investigadores distritales en educación, Bogotá, Serie Memorias, no 1, IDEP, 1997.

Gallardo Cruz, J.; Trianes Torres, M. "Psicología de la educación y del desarrollo”, en Contextos escolares, Barcelona, Editorial Pirámide, 2006.

Gallego Anabitarte, A. "Conceptos y principios fundamentales del derecho de organización”. Lecciones de Derecho Administrativo, no 1, Barcelona, Marcial Pons, 2001.

García Canclini, N. "Ciudades paranoicas miradas desde América Latina”, en Dossier La Vanguardia. América Latina, no 4, Democracia, Neoliberalismo y Populismo, enero-marzo de 2003.

García De Enterría E.; Fernández Rodríguez, T. Curso de Derecho Administrativo, t. I, Madrid, Thompson Civitas, 2006.

García Herrera, M. "Intereses difusos, intereses colectivos y función mediadora”, en Jueces para la Democracia, no 12, 1991.

García Oviedo, C. Derecho Administrativo, vol. I, Madrid, Librería General de Victoriano Suárez, 1943.

Garrido Cordobeira, L. Los daños colectivos y la reparación, Buenos Aires, Universidad, 1993.

Gascón y Marín, J. Tratado de derecho administrativo, t. II, Madrid, Bermejo Impresor, 1955.

Giannini, M. Derecho Administrativo, vol. I, Madrid, Ministerio de Administraciones Públicas, 1991.

Gidi, A. "Derechos difusos, colectivos e individuales homogéneos", en La tutela de los derechos difusos, colectivos e individuales homogéneos. Hacia un código modelo para Iberoamérica, México, Editorial Porrúa, 2004.

Gómez, J. J. Conferencias de Derecho Civil. Bienes, Bogotá, Universidad Nacional de Colombia, 1960.

Gordillo Amado, A. "De los intereses difusos”, tesis de grado, Bogotá, Universidad Externado de Colombia, 1988.

Henao Pérez, J. C. "¿Se defienden de la misma manera los derechos colectivos en derecho colombiano y en derecho francés?”, en IV Jornadas de Derecho Constitucional y Administrativo, Bogotá, Universidad Externado de Colombia, 2003.

Hernández Martínez, M. Mecanismos de tutela de los intereses difusos y colectivos, México, Universidad Nacional Autónoma, 1997.

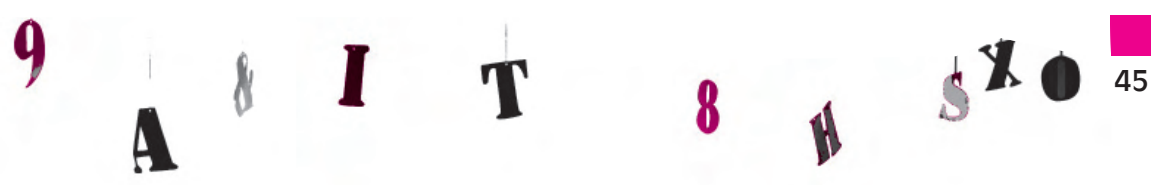


Ortiz, E. Tesis de Derecho Administrativo, t. II, Bogotá, Biblioteca Jurídica DIKE, 2002.

Parada, R. Derecho Administrativo III. Bienes Públicos. Derecho Urbanístico, Barcelona, Marcial Pons, 2007.

Parejo Alfonso, L; Jiménez Blanco, A; Ortega Álvarez, L. Manual de Derecho Administrativo, Barcelona, Editorial Ariel, 1990.

Peces-Barba, G. Curso de derechos fundamentales. Teoría general, Madrid, Universidad Carlos III, 1998.

Pérez Luño, A. "La universalidad de los derechos humanos y el Estado constitucional”, en serie de teoría jurídica y filosófica del Derecho, no 23, Bogotá, Universidad Externado de Colombia, 2002.

Pérez Rollo, F. Derecho financiero y tributario, Madrid, Cívitas, 2001.

Pisciotti Cubillos, D. Los derechos de tercera generación, los Intereses difusos o colectivos y sus modos de protección (acción popular), Bogotá, Universidad Externado de Colombia, 2001.

Restrepo, J. C. Hacienda Pública, Bogotá, Universidad Externado de Colombia, 1998.

Rincón Córdoba, J. I. Las generaciones de los derechos fundamentales y la acción de la administración pública, Bogotá, Universidad Externado de Colombia, 2004.

"La aplicación del artículo 6.1 de la Convención Europea de Derechos Humanos en las actuaciones administrativas y en el control judicial ejercido sobre las actividades de la administración pública”, en Revista de Jurisprudencia Administrativa, no 4, 2003. www.uexternado.edu.co

. "Los derechos colectivos y la acción de la administración pública”, en II Jornadas de Derecho Constitucional y Administrativo, Bogotá, Universidad Externado de Colombia, 2002.

. Derecho Administrativo Laboral (empleo público, carrera administrativa y derecho a la estabilidad en el empleo), Bogotá, Universidad Externado de Colombia (en imprenta).

Rodríguez Meléndez, R. "Intereses y tutela constitucional”, en www.uv.es/ripj/2salva.htm.

Rodríguez Rodríguez, L. Derecho Administrativo general y colombiano, Bogotá, Editorial Temis, 2008.

Saénz Obregón, J. Desconfianza, civilidad y estética: las prácticas formativas estatales por fuera de la escuela en Bogotá 1994 2003, Bogotá, IDEP, 2007.

Santofimio Gamboa, J. O. Tratado de Derecho Administrativo. Introducción, Bogotá, Universidad Externado de Colombia, 2003.

Sayagés Laso, E. Tratado de Derecho Administrativo, t. I-II, Montevideo, Editorial Martín Bianchi Altuna, 1986.

Secretaría de Educación Distrital. Foro educativo distrital 2008, "Evaluación integral para la calidad educativa”, Bogotá.

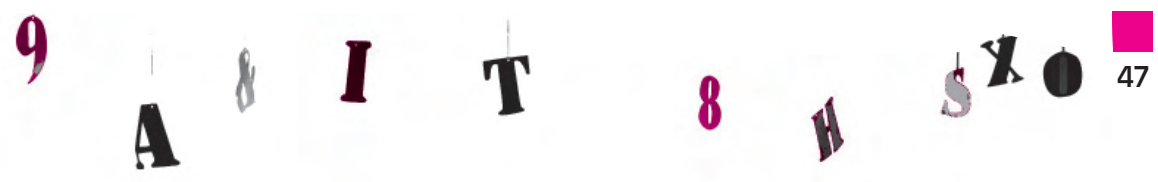




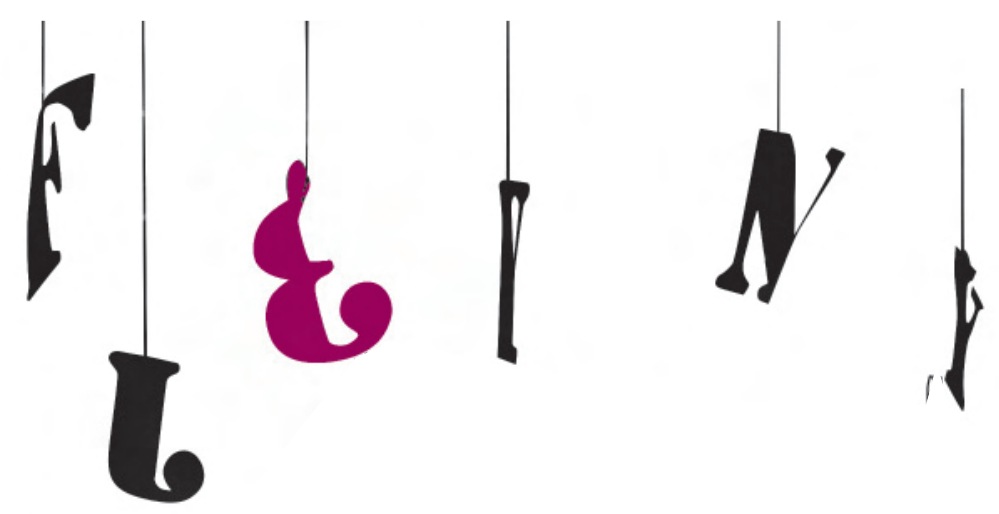

Suárez Fierro, L. "El poder tributario como emblema de la democracia. El principio de legalidad como límite del poder tributario desarrollado jurisprudencialmente”, en Lecturas en Derecho Fiscal, Bogotá, Universidad Externado de Colombia, 2003.

Tobón Tobón, S. Formación basada en competencias, pensamiento complejo, diseño curricular y didáctica, Bogotá, ECOE, 2004.

Trujillo García, S. La sujetualidad: un argumento para implicar. Propuesta para una pedagogía de los afectos, Bogotá, Pontificia Universidad Javeriana, 2008.

Valdés Costa, R. Curso de Derecho Tributario. Ingresos públicos. Normas tributarias. Relación jurídica tributaria, Bogotá, Editorial Temis, 2001.

Valencia Zea, A. Derecho Civil, t. II, Bogotá, Temis, 2007.

Vidal Perdomo, J. Derecho Administrativo, Bogotá, Legis, 2008.

Vigoritti, V. Interessi collettivi e proceso: La Legittimazione ad agire, Milán, Giuffré Editore, 1979.

Villar Borda, L. "Derechos humanos: responsabilidad y multiculturalismo", en Serie de teoría jurídica y filosofía del Derecho, no 9, Bogotá, Universidad Externado de Colombia, 2004.

Villar, L; Rosales, J. M. “Las razones de la convivencia”, en Serie de teoría jurídica y filosofía del Derecho, no 49, Bogotá, Universidad Externado de Colombia, 2007.

Zagrebelsky, G. El Derecho dúctil. Ley, derechos, justicia, Madrid, Trotta, 1995.

Zaneti Junior, H. "Los derechos colectivos lato sensu: la definición conceptual de los derechos difusos, de los derechos colectivos stricto sensu, y de los derechos individuales homogéneos”, en La tutela de los derechos difusos, colectivos e individuales homogéneos. Hacia un código modelo para Iberoamérica, México, Editorial Porrúa, 2004.

Zubiría Samper, J. Los modelos pedagógicos, Fundación Alberto Merani, Bogotá, 1994.

Zuluaga Garcés, O. L. Pedagogía y epistemología, Bogotá, Magisterio, 2003. 